\title{
Coincidência de conexão do tom emocional com abstração e o resultado psicoterapêutico
}

\author{
Coincidence of connecting emotional tone with \\ abstraction and the psychotherapeutic outcome
}

\author{
Elisa Medici Pizão YOSHIDA \\ Erhard MERGENTHALER ${ }^{2}$
}

\begin{abstract}
Resumo
O estudo objetivou testar a afirmação de que "pacientes que não têm êxito em conectar suas emoções com cognição durante suas terapias provavelmente não melhorarão". Dois processos de psicoterapias psicodinâmicas breves, um bem-sucedido e outro malsucedido, foram analisados com o Modelo dos Ciclos Terapêuticos. O Modelo dos Ciclos Terapêuticos é um método de análise de texto por computador que permite identificar padrões de emoção-abstração na narrativa de interlocutores. Quatro padrões são identificados: Relaxamento, Experiência, Reflexão e Conexão. Momentos clinicamente significantes são mais associados à Conexão. Levantou-se a hipótese de que o caso bem-sucedido apresentaria maior proporção de Conexão. Quanto à Reflexão, ao Relaxamento e à Experiência, não eram esperadas diferenças. Encontrou-se maior proporção de Conexão e de Relaxamento no caso bem- sucedido, e diferenças não significantes em Reflexão e Experiência. Considerações sobre a magnitude das mudanças, valência das emoções e abstrações e sequência de ocorrência dos padrões sugerem novas análises.
\end{abstract}

Unitermos: Evidência empírica. Padrões de emoção abstração. Pesquisa de processo psicoterapêutico.

\begin{abstract}
The current study aimed to test the statement that "patients who do not succeed in connecting their emotions with cognition during therapy are unlikely to improve". Two processes of brief psychodynamic psychotherapies, one successful the other not, were analyzed in accordance with the Therapeutic Cycles Model. The Therapeutic Cycles Model is a computer-assisted text analysis method that permits the identification of emotion-abstraction patterns in narratives by interlocutors. Four patterns are identified: Relaxing, Experiencing, Reflecting and Connecting. Clinically significant moments are mostly associated with Connecting. It was hypothesized that a successful process would differ from an unsuccessful one, having a higher proportion of Connecting. As far as Reflecting, Experiencing and Relaxing are concerned, no differences were expected. Results pointed to a higher proportion of Connecting and Relaxing in the successful process and no difference in respect of Experiencing and Reflecting. Considerations about the magnitude of change, emotion and abstraction valence and the sequence of patterns, suggest further analysis.
\end{abstract}

Uniterms: Empirical evidence. Emotion-abstraction patterns. Research on psychotherapeutic process.

arer

1 Pontifícia Universidade Católica de Campinas, Centro de Ciências da Vida, Programa de Pós-Graduação em Psicologia. Av. Jonh Boyd Dunlop, s/n., Prédio Administrativo, Jd. Ipaussurama, 13090-950. Campinas, SP, Brasil. Correspondência para/Correspondence to: E.M.P. YOSHIDA. E-mail: $<$ eyoshida.tIn@terra.com.br>.

2 Ulm University. Ulm, Alemanha.

Apoio: O projeto de pesquisa recebeu financiamento das agências Fapesp/Daad (Linha de Fomento Auxílio visitante exterior - Processo 2008/05056-6). 
São apresentados, neste artigo, os resultados de um estudo exploratório cujo objetivo foi testar empiricamente a afirmação de que "pacientes que não têm êxito em conectar suas emoções com cognição durante suas terapias provavelmente não melhorarão" (Mergenthaler, 2008, p.113), este constitui um dos pressupostos em que se baseia o Modelo dos Ciclos Terapêuticos (TCM) (Mergenthaler, 1996) para explicar a ocorrência de mudança em processos de psicoterapia.

O TCM é um método de análise de textos por computador desenvolvido com o propósito de auxiliar a identificação de momentos-chave em processos de psicoterapias. Momentos-chave são caracterizados pela ocorrência de progresso na resolução do problema que levou o paciente à psicoterapia. São identificados pela ocorrência de marcadores linguísticos associados à expressão de emoções e de abstrações (Mergenthaler, 1996, 2008).

Considera-se que a expressão de emoções é um fator central de todo e qualquer processo terapêutico e que a ela deve estar associada a capacidade de perceber e refletir sobre a experiência vivida e de expressá-la verbalmente. Nessa medida, também é relevante a existência de habilidades cognitivas que permitam ao paciente refletir sobre sua experiência emocional (Lepper \& Mergenthaler, 2007). Emoções e abstrações são consideradas necessárias para a ocorrência de mudança em processos psicoterapêuticos e se encontram diretamente relacionadas à qualidade dos resultados obtidos (Fontao de Ross \& Mergenthaler, 2005).

Mergenthaler (1996, p.1306) propõe o conceito de tom emocional para designar a "densidade das palavras de emoção em um dado texto". Pode-se, portanto, falar em baixa ou alta densidade de emoção, ou, simplesmente, alta e baixa emoção. O mesmo se aplica a abstrações, em que baixa ou alta abstração corresponde à densidade da abstração (Mergenthaler, 1996; 2008). A medida da densidade das emoções e abstrações é dada pela frequência de palavras associadas a cada uma dessas categorias em cada momento da sessão. A ideia é que a proeminência de um determinado tema possa ser aferida pelo número de palavras utilizadas para descrevê-lo. A quantidade de palavras permite, portanto, uma avaliação qualitativa que se traduz em padrões de emoção-abstração (Mergenthaler, 1996; 1998). Com base

36 nas categorias de baixa e alta densidade para emoções e abstrações, foram propostos quatro padrões clinicamente relevantes: Relaxamento, Reflexão, Experiência e Conexão (Mergenthaler, 1996).

O padrão Relaxamento está associado a baixo tom emocional e baixa abstração; o paciente fala sobre um material que não está manifestamente conectado aos seus sintomas ou temas centrais. Isso costuma ocorrer nos momentos iniciais do processo terapêutico ou após a abordagem de temas sensíveis e permite que o paciente se recupere física e psiquicamente antes de dar continuidade ao seu processo.

O padrão Reflexão é caracterizado por baixo tom emocional e alta abstração; o paciente recorre a abstrações sem utilizar expressões com conteúdo emocional. Acontece nos momentos em que ele está refletindo sobre alguma experiência afetiva relevante ou, ao contrário, quando precisa esquivar-se de temas e experiências emocionalmente perturbadores que ponham em risco seu equilíbrio interno. Nesse último caso, o padrão Reflexão tem uma função defensiva, usualmente identificada como racionalização (Mergenthaler, 2008).

O padrão Experiência corresponde à alta emoção e à baixa abstração. O paciente aborda tema conflituoso ou que enseje a experiência de emoções, sem que seja capaz de elaborá-las cognitivamente.

A ocorrência simultânea de alto tom emocional e alta abstração caracteriza o padrão Conexão: a expressão da emoção é acompanhada de trabalho cognitivo que permite ao paciente refletir sobre seus conflitos. Indica, portanto, um momento relevante ou momento-chave do processo, em que a ocorrência de mudança pode ser mais claramente identificada (Mergenthaler, 1996; 2008).

Esses padrões podem ser representados graficamente por meio de uma combinação de escores-Z das frequências relativas para tom emocional e abstração (Figura 1) e permitem o acompanhamento da modulação das emoções e abstrações ao longo do processo e dentro de cada sessão, sendo de especial interesse os momentos correspondentes aos "ciclos terapêuticos" (Mergenthaler, 1996).

O conceito de ciclo terapêutico decorre do pressuposto de que "no curso de uma psicoterapia ou em uma sessão de psicoterapia, os padrões de emoção-abstração não ocorrem por acaso; ao contrário, pre- 
sume-se um processo periódico para as variáveis subjacentes de tom emocional e de abstração" (Mergenthaler, 1996, p.1308). Um modelo prototípico de ciclo terapêutico pressupõe a ocorrência dos padrões de emoção abstração segundo uma sequência temporal de cinco fases:

Fase l: inicia-se com o padrão Relaxamento e corresponde aos momentos em que a pessoa descreve episódios de sua vida, ou se refere a temas com baixa conotação afetiva. A narrativa pode revelar tanto um estado de relaxamento quanto de desorientação, em que a pessoa parece não saber ao certo sobre o que falar (Buchheim \& Mergenthaler, 2000). Prevalecem palavras com baixo Tom Emocional (TE) e Baixa Abstração $(A B)$.

Fase Il: à medida que a pessoa começa a falar de seus sintomas e de suas dificuldades, aumenta a chance de verbalização com conteúdo emocional e, portanto, do aparecimento do padrão Experiência. A pessoa refere-se a situações difíceis com forte conotação afetiva, sem ainda poder elaborá-las cognitivamente. Prevalece o uso de palavras com alto TE e baixa AB.

Fase III: é marcada pela associação de expressão verbal das emoções, acompanhada da possibilidade de refletir sobre elas. O que caracteriza, portanto, o padrão Conexão. Teoricamente, essa fase pode refletir um momento de elaboração e estar associada a um insight (Buchheim \& Mergenthaler, 2000). Observam-se palavras com alto TE e alta $A B$.

Fase IV: como consequência do entendimento e da nova compreensão, favorecidos pelo insight, um momento de reflexão sobre a experiência vivida costuma ocorrer, marcando portanto uma fase caracterizada pela ocorrência do padrão Reflexão, que se traduz por alta $A B$ e baixo TE.
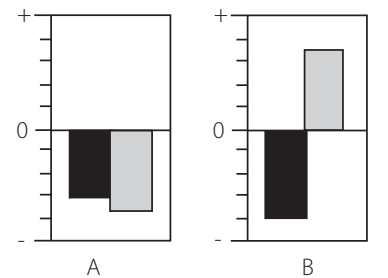

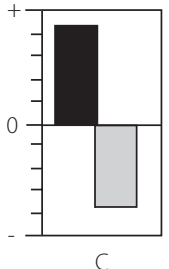

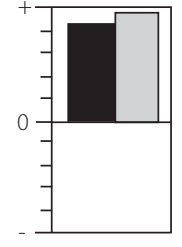

Figura 1. Representação gráfica dos padrões emoção-abstração em escores- $Z$.

Nota: A: Relaxamento; B: Reflexão; C: Experiência; D: Conexão.
Fase V: marca o final do ciclo, com o retorno a um estado de Relaxamento e a preparação para um novo ciclo; apresenta, portanto, baixo TE e baixa AB (Mergenthaler, 1996).

A avaliação empírica do TCM é realizada por meio do software Cycles Model (CM), baseado em listas de palavras que denotam emoção e abstração e que são chamadas de dicionários. Há versões de dicionários em alemão, inglês, italiano, espanhol e em português do Brasil. Em cada caso, uma ampla variedade de textos foi analisada e os marcadores de tom emocional e de abstrações selecionados (Lepper \& Mergenthaler, 2007). Para tanto, as palavras são categorizadas em: emoção positiva (1), emoção negativa (2), abstração (3), ambas, positiva e abstração (4), ambas, negativa e abstração (5).

São exemplos de palavras da categoria emoção positiva (1) acalmar, aceitar, acolher e suas respectivas conjugações; exemplos de palavra de tom emocional negativo (categoria 2) seriam afastar, agitado, adoecer, briga ou brigar; a categoria abstração (3) é integrada, por exemplo, por adolescência, aparência, conhecimento; a categoria de abstrações positivas (4) é integrada por palavras como consideração, coragem, desarmamento, dom; e a quinta categoria, composta por abstrações com tom negativo, pode ser exemplificada por egoísmo, erro, fardo e hostilidade. Como se pode ver, as palavras são categorizadas em função da conotação das ideias ou temas a elas associadas, sendo utilizadas, em uma sessão de psicoterapia, para expressar as vivências emocionais e a experiência cognitiva do paciente. A definição do padrão de emoção-abstração - Relaxamento, Experiência, Reflexão ou Conexão - é dada pela prevalência dessas categorias em cada bloco de palavras analisadas.

No presente estudo, dois processos de psicoterapias psicodinâmicas breves, um bem-sucedido e outro malsucedido, foram analisados com a versão em português dos dicionários (Yoshida, 2009) e os dados comparados de acordo com as seguintes hipóteses: 1) O paciente com resultado bem-sucedido apresenta uma maior proporção de padrão Conexão (alta frequência de palavras emocionais e de abstração) quando comparado ao considerado malsucedido; 2) Quanto aos padrões, Reflexão (alta frequência de palavras de abstração), Experiência (alta frequência de palavras emocionais) e Relaxamento (baixa frequência de palavras de abstração e emocionais), não são esperadas diferenças significantes. 


\section{Método}

\section{Participantes}

O processo terapêutico considerado malsucedido foi identificado como Caso X e o bem-sucedido como Caso Y.

Caso X: Mulher, 50 anos, amasiada, ensino fundamental incompleto, do lar, dez filhos, com medicação antidepressiva, encaminhada à clínica-escola por psiquiatra.

Caso Y: Mulher, 48 anos, desquitada, ensino médio completo, afastada do serviço por doença, três filhos casados, diabética e com medicação antidepressiva, encaminhada à clínica-escola por psiquiatra.

\section{Instrumento}

Modelo dos Ciclos Terapêuticos (Mergenthaler, 1996; 2008) é um método de análise de textos por computador que permite identificar momentos-chave na narrativa do paciente, do terapeuta, ou de ambos, em interação. Com o auxílio do software CM, as palavras de tom emocional positivo ou negativo e as abstrações são identificadas e gráficos dos padrões de emoção-abstração predominantes, em cada segmento da sessão, são gerados. Apesar de ser possível escolher o tamanho do segmento de cada sessão, o mais usual são os blocos de 150 palavras. O conjunto de gráficos gerados com o auxílio do software CM é composto por uma primeira folha com os gráficos correspondentes à ocorrência de marcadores verbais de emoção e de abstração por sessão e identificado como Corpus Overview, e as folhas seguintes trazem os gráficos de cada uma das respectivas sessões (Figura 2).

Em cada folha, o gráfico superior (Language Patterns Patient) fornece os valores em escores- $Z$ das frequências relativas de marcadores utilizados pelo paciente. As barras em preto indicam a frequência de marcadores com tom emocional e os em cinza os de abstração. A linha cinza, que corta o gráfico, indica o movimento médio usando metade do bloco de palavras. Com isso, obtém-se uma progressão "suavizada" (smoothed) do padrão de linguagem do paciente. O segundo gráfico (Speech Proportion) fornece uma ideia da contribuição independente do paciente e do terapeuta, por meio da proporção de palavras de cada um. A contribuição do paciente aparece nas barras pretas, acima do eixo, e as do psicoterapeuta nas barras cinzas, abaixo do eixo. O terceiro gráfico traz essas contribuições integradas. E, finalmente, a valência das emoções é apresentada no gráfico ao final da página, respectivamente, pelas linha cinza (tom emocional positivo) e linha preta (tom negativo). A identificação dos ciclos terapêuticos pode ser feita manualmente ou pelo próprio software. Um ciclo terapêutico é representado por uma sequência de padrões de emoção-abstração, que inclui ao menos um bloco com o padrão Conexão e tem como limite inicial e final a ocorrência de um padrão Relaxamento. Quando não há um padrão Relaxamento anterior ao bloco em que ocorreu Conexão, o início da sessão é considerado o início do ciclo (Lepper \& Mergenthaler, 2008).

\section{Procedimentos}

As participantes foram selecionadas de uma amostra de quatro pacientes de estudo anterior, cujo objetivo foi o de avaliar aspectos do resultado e do processo de psicoterapias psicodinâmicas breves, conduzidas por terapeutas experientes (o Caso X foi atendido por psicoterapeuta do sexo masculino, com 35 anos de experiência clínica; e o Caso Y por psicoterapeuta do sexo feminino, com 33 anos de experiência). As sessões foram gravadas em áudio e vídeo e posteriormente

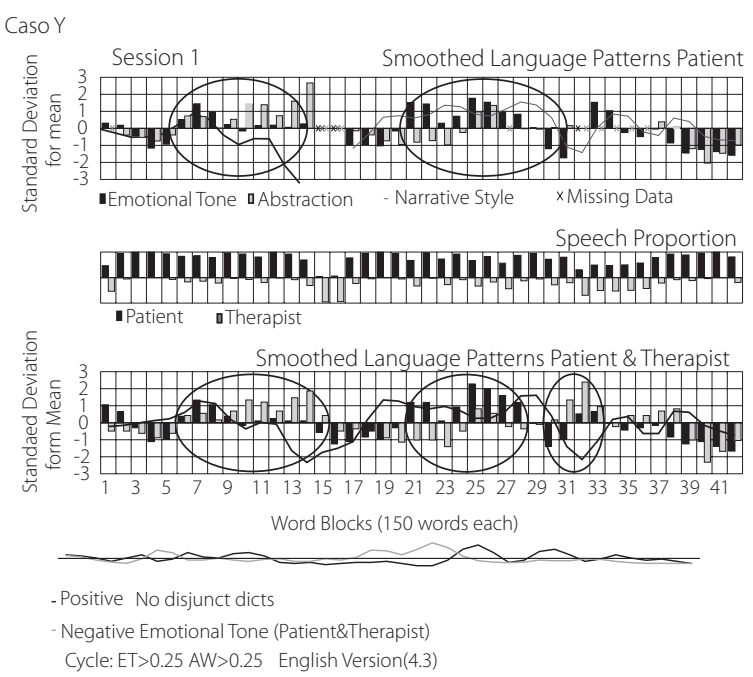

Figura 2. Modelos dos Ciclos Terapêuticos: primeira sessão Caso Y. 
transcritas. O projeto de pesquisa foi aprovado pelo Comitê de Ética em Pesquisas com Seres Humanos da Pontifícia Universidade Católica de Campinas (PUC-Campinas) (Protocolo: 353/04) (Yoshida, 2008a).

$\mathrm{Na}$ avaliação de variáveis de processo foram utilizados: a Escala de Avaliação dos Mecanismos de Defesa (DMRS) (Perry,1990), Intervenções do Terapeuta (IT) (Gabbard, 1992) e o método do Tema Central de Relacionamento Conflituoso (CCRT) (Luborsky \& Crits-Christoph, 1998). Para a avaliação de resultados, foram obtidas medidas de autorrelato no início, na fase medial, final e em entrevistas de seguimento, entre três e seis meses após o término das psicoterapias. Foram utilizados o Inventário de Depressão Beck (BDI) (Cunha, 2001), a Versão em Português da Escala de Alexitimia de Toronto (TAS) (Yoshida, 2000), a Escala Fatorial de Ajustamento Emocional/ Neuroticismo (EFN) (Hutz \& Nunes, 2001) e a Escala de Avaliação de Sintomas-40 (EAS-40) (Laloni, 2001).

O resultado, ao final da psicoterapia e nas entrevistas de seguimento, foi confrontado com o do início do processo para cada uma das medidas de autorrelato. Utilizou-se o critério de "mudança clinicamente significante", proposto por Jacobson e Truax (1991), de acordo com o qual, ao final da psicoterapia, o paciente deve apresentar desempenho equivalente ao de população funcional em relação à variável medida, além da magnitude da mudança ter sido suficientemente grande para ser atribuída a uma mudança "real", e não a erros de medida. A implementação desses critérios foi feita com base nos pontos de corte e na magnitude de mudança confiável correspondente a um $\mathrm{RCl}=1,96[\mathrm{RCl}$, sigla em inglês para Índice de Mudança Confiável (Jacobson \& Truax, 1991)], conforme sugerido por Yoshida (2008b). São os seguintes os valores de corte: $\mathrm{BDI}=12$ [coincide com o ponto de corte apontado por Cunha (2001), com base em amostra normativa]; TAS = 73 [coincide com o ponto de corte internacionalmente aceito (Taylor, Bagby \& Parker, 1992)]; EAS-40 (total) $=1$; EAS-40 (F1) =1; EAS-40 $(F 2)=1 ; \quad E A S-40(F 2)=1 ; E A S-40(F 4)=1 ; E F N 1=86 ;$ $\mathrm{EFN} 2=32 ; \mathrm{EFN3}=90 ; \mathrm{EFN} 4=52$.

Quanto aos valores da magnitude de mudança confiável de cada medida, correspondem respectivamente os seguintes valores: $\mathrm{BDI}=12 ; \mathrm{TAS}=15$; EAS-40 $($ Total $)=0,30 ;$ EAS-40 (F1) =0,52; EAS-40 (F2) =0,44; EAS-40 $(F 3)=0,42 ; \mathrm{EAS}-40(F 4)=0,34 ; \mathrm{EFN} 1=22 ; \mathrm{EFN2}=7$;
EFN3 =21; EFN4=14 (Yoshida, 2008b). Para a definição do status de mudança, também se adotaram os critérios sugeridos por Jacobson e Truax (1991). Escores superiores ao ponto de corte corresponderam a "recuperação", e uma diferença entre os escores do pré e pós-tratamento, superior ao valor reportado para a magnitude de mudança confiável da medida, indica "melhora" na variável avaliada (Yoshida, 2008b). Quando cada critério foi atingido, a mudança foi indicada por um S (sim). E, nos casos negativos, por um N (não). No caso em que a participante já se encontrava na faixa de funcionalidade, por ocasião do início da psicoterapia, o status de mudança foi indicado por $M$.

No presente estudo, foram utilizadas apenas as medidas de resultado para definir quais teriam sido os processos terapêuticos com melhor e com pior resultado. Os resultados da fase inicial e final das psicoterapias, assim como o status de mudança das participantes com melhor (Caso Y) e pior (Caso X) desempenhos, são apresentados na Tabela 1. Esses resultados já foram apresentados em artigos anteriores, em que outros aspectos da pesquisa foram analisados. Os resultados do Caso Y foram publicados em Yoshida et al. (2009), e os do Caso X, em Yoshida (2008b). Definidos os dois processos terapêuticos, as respectivas transcrições das sessões foram analisadas com o TCM e as hipóteses de pesquisas testadas.

\section{Resultados}

A Tabela 2 traz a distribuição dos padrões emoção-abstração de cada um dos processos psicoterapêuticos, segundo cada sessão e no total. A última coluna fornece indicação da frequência relativa de cada padrão.

O teste das hipóteses foi feito utilizando-se o teste de Qui-quadrado para amostras independentes. Os resultados indicaram que não houve diferença significante na proporção de padrões de emoção-abstração entre os dois casos $\left[\chi^{2}(3, N=776)=3,36\right.$, $p>0,20]$. A paciente do Caso Y apresentou proporção significantemente mais elevada do padrão Conexão $\left.\left[\chi^{2}(1, N=157)=7,80, p<0,01\right)\right]$ e do padrão Relaxamento $\left[\chi^{2}(1, N=249)=9,64, p<0,01\right]$. Em relação aos padrões Experiência $\left[\chi^{2}(1, N=185)=1,95, p>0,10\right]$ e Reflexão $\left[\chi^{2}(1, N=185)=0,65, p>0,30\right]$, as diferenças não foram significativas. 
Tabela 1. Escore inicial, final e status de mudança no BDI,TAS, EFN e EAS-40.

\begin{tabular}{|c|c|c|c|c|c|c|c|c|}
\hline \multirow[b]{2}{*}{ Instrumento } & \multicolumn{4}{|c|}{ Caso $\mathrm{Y}$} & \multicolumn{4}{|c|}{ Caso $X$} \\
\hline & Inicial & Final & Melhorou(RCl>1,96) & $\begin{array}{l}\text { Recuperou-se } \\
\text { (funcional) }\end{array}$ & Inicial & Final & Melhorou $(\mathrm{RCl}>1,96)$ & $\begin{array}{l}\text { Recuperou-se } \\
\text { (funcional) }\end{array}$ \\
\hline BDI & 3 & 14 & $S$ & $\mathrm{~N}$ & 37 & 24 & $S$ & $N$ \\
\hline TAS & 70 & 64 & $\mathrm{~S}^{1}$ & S & 73 & 49 & $S^{1}$ & S \\
\hline $\mathrm{EFN} 1^{3}$ & 90 & 51 & S & S & 107 & 113 & N & N \\
\hline EFN2 & 14 & 17 & $M^{2}$ & M & 18 & 14 & $M^{2}$ & M \\
\hline EFN3 & 141 & 123 & $\mathrm{~N}$ & $\mathrm{~N}$ & 117 & 106 & $N$ & $N$ \\
\hline EFN4 & 58 & 27 & S & S & 87 & 86 & N & N \\
\hline EAS-40T & 1,08 & 0,55 & S & S & 1,68 & 1,28 & S & N \\
\hline$F 1^{4}$ & 0,90 & 0,10 & S & S & 1,50 & 0,90 & s & S \\
\hline $\mathrm{F} 2$ & 0,80 & 0,40 & M & M & 1,90 & 1,50 & N & N \\
\hline F3 & 1,40 & 1,10 & N & N & 1,60 & 1,70 & N & $N$ \\
\hline $\mathrm{F}_{4}$ & 1,20 & 0,60 & S & S & 1,70 & 1,0 & S & S \\
\hline
\end{tabular}

${ }^{1}$ Encontrava-se na faixa de funcionalidade no início do atendimento; ${ }^{2} \mathrm{M}$ : Mudança não esperada (desajuste psicossocial); ${ }^{3}$ EFN1: vulnerabilidade; EFN2: desajuste psicossocial; EFN3: instabilidade/ansiedade; EFN4: depressão; ${ }^{4}$ F1: psicotismo; F2: obsessividade-compulsividade; F3: somatizaçăo; F4: ansiedade.

Tabela 2. Distribuição de padrões emoção-abstração do Caso Y e Caso X.

\begin{tabular}{|c|c|c|c|c|c|c|c|c|c|c|c|c|c|c|}
\hline & Sessão & 1 & 2 & 3 & 4 & 5 & 6 & 7 & 8 & 9 & 10 & 11 & $\Sigma$ & $f \%$ \\
\hline \multirow{6}{*}{$\begin{array}{l}> \\
\text { o } \\
\ddot{U}\end{array}$} & Padrão & & & & & & & & & & & & & \\
\hline & Relaxamento & 13 & 18 & 15 & 14 & 12 & 14 & 16 & 8 & 15 & 14 & 10 & 149 & 33,48 \\
\hline & Experiência & 8 & 11 & 11 & 11 & 13 & 17 & 6 & 5 & 10 & 7 & 3 & 102 & 22,92 \\
\hline & Conexão & 12 & 6 & 13 & 7 & 12 & 4 & 5 & 3 & 16 & 10 & 8 & 96 & 21,57 \\
\hline & Reflexão & 8 & 8 & 11 & 13 & 10 & 8 & 12 & 5 & 8 & 8 & 7 & 98 & 22,09 \\
\hline & $\Sigma$ & 41 & 43 & 50 & 45 & 47 & 43 & 39 & 21 & 49 & 39 & 28 & 445 & 100,0 \\
\hline \multirow{5}{*}{$\begin{array}{l}\times \\
0 \\
\mathscr{J}\end{array}$} & Relaxamento & 1 & 8 & $*$ & 11 & 9 & 13 & 15 & 16 & 10 & 11 & 6 & 100 & 30,21 \\
\hline & Experiência & 5 & 9 & & 12 & 11 & 9 & 12 & 3 & 6 & 11 & 5 & 83 & 25,07 \\
\hline & Conexão & 1 & 10 & & 7 & 7 & 9 & 6 & 9 & 5 & 4 & 3 & 61 & 18,43 \\
\hline & Reflexão & 6 & 15 & & 6 & 8 & 8 & 10 & 9 & 9 & 11 & 5 & 87 & 26,28 \\
\hline & $\Sigma$ & 13 & 42 & & 36 & 35 & 39 & 43 & 30 & 30 & 37 & 19 & 331 & 100,0 \\
\hline
\end{tabular}

*3a sessão-inaudível.

\section{Discussão}

A ausência de diferença significante na proporção de padrões de emoção-abstração $(p>0,20)$ sugere que ambas as pacientes estiveram ativamente empenhadas nos respectivos processos psicoterapêuticos, expressando-se verbalmente e interagindo com seus psicoterapeutas. Pode-se, então, dizer que os processos são comparáveis, no que diz respeito às unidades de análises com o TCM.

Os resultados corroboraram a expectativa de que o caso mais bem-sucedido apresentaria maior proporção de Conexão $(p<0,01)$. A maior proporção de 40 Conexão no Caso Y, quando comparada ao Caso $X$, sugere que um trabalho mais consistente para a superação dos problemas teria sido efetivamente realizado com a expressão verbal das emoções acompanhada de palavras com conotação abstrata, indicativas de trabalho intelectual em torno das emoções.

De acordo com o esperado, não houve diferenças significantes entre os dois casos em relação às proporções do padrão Reflexão $(p>0,30)$ e do padrão Experiência $(p>0,10)$. O padrão Reflexão é usualmente visto como indicativo de um momento que sucede ao insight devido a uma nova compreensão da situação (Mergenthaler, 1996). Seria um momento em que o paciente discute aspectos relacionados às suas dificuldades de forma menos emocional e mais cognitiva, 
como um prolongamento da fase de elaboração. Contudo, em outras situações, o padrão Reflexão pode desempenhar um papel defensivo ao impedir o afloramento, à consciência do paciente, de conteúdos de forte tom emocional, capazes de desestabilizar seu equilíbrio. Observa-se, nesses momentos, a ocorrência da defesa intelectualização (Mergenthlaer, 2008), que "envolve a substituição de uma razão plausível para uma determinada ação ou impulso por parte do sujeito, quando um motivo que é mais de interesse pessoal ou de difícil reconhecimento está evidente para o observador" (Perry, 1990, p.25). Ou seja, apesar de as pacientes não se diferenciarem quanto à proporção do padrão Reflexão, seu papel, em cada processo, pode ser diferente. Nesse sentido, é possível levantar a hipótese de que, no Caso Y, os conteúdos abordados nos momentos de Reflexão tenham colaborado para a maior proporção de Conexão, o que, por sua vez, pode ter ensejado melhores resultados. No Caso X, o padrão Reflexão teria tido uma função mais defensiva, protegendo a paciente de níveis intoleráveis de sofrimento psíquico. Essas hipóteses parecem plausíveis apenas com base na compreensão clínica dos casos. Todavia, para o esclarecimento efetivo da função do padrão Reflexão, em cada um dos casos, faz-se necessário o retorno ao texto e à análise qualitativa da verbalização.

Quanto ao padrão Experiência, as pacientes teriam se detido em proporções semelhantes na narração de situações com forte conotação afetiva. Momentos dominados por emoções negativas são intitulados por Mergenthler (2008) de "aprofundar e suprir" (deepen-and-provide), e, na expressão de emoções positivas, como decorrência da melhora do paciente, de "alargar e construir" (broaden-and-build). Ambos os movimentos são necessários para a superação das dificuldades, que deve, todavia, ser acompanhada do trabalho reflexivo associado ao padrão Conexão. Para compreender mais profundamente qual o papel desempenhado pelo padrão Experiência, em cada caso, seria necessário levar em conta a valência das emoções e abstrações verbalizadas (positivas e negativas) nesses segmentos e verificar se, de fato, no Caso Y, eles foram mais frequentemente acompanhados de Conexão.

Contrariamente ao esperado, o Caso Y apresentou uma maior proporção do padrão Relaxamento $(p<0,01)$. Uma análise detalhada do gráfico do TCM também deve fornecer alguma compreensão para isso. A posição relativa desse padrão em relação aos padrões Conexão e Reflexão deve fornecer pistas quanto à "função" do Relaxamento a cada momento: se ele teria tido mais uma função de permitir à paciente $Y$ se recuperar física e psiquicamente antes de dar continuidade ao seu processo (Mergenthaler, 2008) ou se, ao contrário, teria se caracterizado principalmente por situações de pouco envolvimento no processo, como se ela não soubesse ao certo sobre o que falar (Buchheim \& Mergenthaler, 2000).

O exame dos resultados das medidas de autorrelato pode complementar as análises com o TCM na medida em que fornece informações sobre algumas das condições com que cada participante enfrentou o processo terapêutico. Ambas as participantes iniciaram os processos psicoterapêuticos com índices elevados de sintomas depressivos no BDI (Tabela 1), sendo moderado (33) no Caso Y e severo (37) no caso X. Em relação ao final do processo, ambas apresentaram melhora clinicamente significante $(\mathrm{RCl}>1,96)$, ainda que nenhuma tenha atingido o nível considerado funcional, isto é, tenha tido escore abaixo do ponto de corte correspondente ao escore 12.

Em relação à alexitimia (TAS), apesar de ambas as pacientes já se encontrarem dentro dos limites esperados para populações não clínicas no início dos atendimentos ( $\leq 73)$, elas apresentaram níveis ainda mais baixos ao final do processo, satisfazendo o critério de mudança confiável. Quanto às medidas de neuroticismo/estabilidade (EFN), a paciente $Y$ iniciou o processo psicoterapêutico com escores acima dos pontos de corte nas dimensões, vulnerabilidade (EFN1>86), instabilidade/ ansiedade (EFN3 >90) e depressão (EFN4 >52), tendo evoluído para o nível funcional nas dimensões, vulnerabilidade (EFN1) e depressão (EFN4), mas mantinha índice elevado de instabilidade/an-siedade (N3) ao final do processo. Quanto à dimensão desajustamento/ emocional, já estava dentro do esperado para populações não clínicas (<32) no início e se manteve nessa faixa até o término (Tabela 1).

Para a paciente $X$, não foram observadas mudanças clinicamente significantes em nenhuma das dimensões de neuroticismo avaliadas por meio da EFN (Tabela1), com exceção da dimensão desajustamento 
psicossocial (EFN3), que já se encontrava dentro dos limites de funcionalidade no início do processo. Como para a paciente $Y$, não havia motivos para esperar que ela apresentasse melhora nessa dimensão, usualmente associada a comportamentos sexuais de risco, consumo exagerado de álcool, hostilidade com pessoas ou animais, necessidade de chamar a atenção, manipulação e descaso de regras sociais (Hutz \& Nunes, 2001).

Quanto ao nível de sintomas psicopatológicos avaliados com a EAS-40, a paciente $Y$ iniciou o atendimento com escores compatíveis aos de população funcional ( $<1)$ em psicoticismo (F1) e obsessividade/compulsividade ( F2), mas foi considerada disfuncional em relação ao escore total da escala e nas dimensões somatização (F3) e ansiedade (F4). Ao final do processo, demonstrou melhora clinicamente significante em todas as dimensões, com exceção da somatização (F3), provavelmente devido às complicações no seu quadro de diabetes, com vários sintomas físicos e sofrimento psíquico evidentes.

A paciente $X$ apresentava níveis elevados de sintomas nas quatro dimensões da EAS-40 no início do processo e, ao final, demonstrava melhora clinicamente significativa nas dimensões psicoticismo e ansiedade, cujo escore correspondia ao ponto de corte (1). A dimensão psicoticismo avalia sintomas relacionados à hostilidade e ideias paranóides (Laloni, 2001), estando usualmente presentes em pessoas com transtornos graves de personalidade, o que não correspondia à situação da paciente. A dimensão ansiedade avalia sintomas de ansiedade generalizada, ansiedade fóbica, direcionada a objetos específicos (Laloni, 2001), o que parece ter sido trabalhado de forma bem-sucedida ao longo do processo. os processos foram observadas mudanças de natureza sintomatológica, como as avaliadas por meio do BDI e da EAS-40, e também mudanças relacionadas a traços mais duradouros de personalidade, tais como o nível de alexitimia (TAS) e de neuroticismo (EFN). Apesar de, no caso $Y$, as mudanças terem sido mais amplas, com o retorno à funcionalidade em um número maior de dimensões avaliadas, cabe assinalar que nenhuma das participantes havia atingido o nível de funcionalidade nos sintomas de depressão (BDI), instabilidade/ansieda-

42 de (EFN3) e somatização (EAS-40-F4) ao final do processo.
Ou seja, apesar de, em alguns aspectos, a paciente $Y$ ter apresentado mais progresso, ela ainda apresentava, ao final do processo, sintomas de depressão, de instabilidade e de somatização em níveis compatíveis com os de populações clínicas. Por outro lado, não se pode dizer que o Caso $X$ teria sido totalmente malsucedido. Quando se consideram a severidade dos sintomas psicopatológicos do início da psicoterapia e a tendência de queda para a maioria das medidas, parece que o resultado insatisfatório se deve mais ao término prematuro do atendimento do que propriamente à falta de empenho da paciente. Pacientes com sintomatologia mais severa necessitam efetivamente de um acompanhamento mais prolongado (Yoshida \& Rocha, 2007).

\section{Considerações Finais}

Pode-se dizer que os dados corroboraram parcialmente as hipóteses levantadas. Apoiaram a principal expectativa teórica de que o caso mais bem-sucedido deveria apresentar uma maior proporção do padrão Conexão. Também confirmaram a expectativa de que não haveria diferenças significantes nas proporções dos padrões Reflexão e Experiência. Quanto ao padrão Relaxamento, ao contrário do esperado, a proporção foi maior no caso bem-sucedido.

Apesar de os resultados terem, em sua maioria, corroborado as hipóteses teóricas, é preciso ressaltar que, clinicamente, o Caso Y não teria sido muito mais bem-sucedido do que o Caso X. Ademais, as análises da ocorrência dos padrões de emoção-abstração, de acordo com oTCM, sugeriram a necessidade de aprofundamento das investigações, contemplando, dessa maneira, os aspectos qualitativos dos processos. É preciso notar as valências das emoções associadas ao padrão Experiência. Em relação ao padrão Reflexão é necessário compreender o papel que ele teria desempenhado em cada caso: se teria ensejado a elaboração de novas sínteses e compreensões por parte do paciente ou se, ao contrário, teria tido uma função defensiva, evitando o afloramento de conteúdos latentes. Da mesma forma, compreender o papel do padrão Relaxamento no caso bem-sucedido pode contribuir para significar melhor sua contribuição para a ocorrência do padrão Conexão.

As análises sugerem que as hipóteses levantadas não teriam contemplado adequadamente a comple- 
xidade dos fatores envolvidos no desfecho de processos psicoterapêuticos ao se cingirem apenas ao aspecto quantitativo da ocorrência dos padrões. Dito de outra forma: análises baseadas exclusivamente na proporção de padrões de emoção-abstração parecem ser insuficientes para identificar diferenças entre processos de psicoterapia, especialmente quando os resultados clínicos não são tão diferentes. Estudos envolvendo psicoterapias realmente bem-sucedidas e malsucedidas podem eventualmente fornecer resultados mais conclusivos. Finalmente, hipóteses considerando a valência das emoções e das abstrações, a sequência na qual os padrões de emoção-abstração ocorrem, assim como considerações sobre o momento de sua ocorrência no processo psicoterapêutico, devem ser testadas em etapas futuras desta pesquisa.

\section{Referências}

Buchheim, A., \& Mergenthaler, E. (2000). The relationship among attachment representation, emotion-abstraction patterns, and narrative style: a computer-based text analysis of the Adult Attachment Interview. Psychotherapy Research, 10 (4), 390-407.

Cunha, J. A. (2001). Manual daversão em português das escalas Beck. São Paulo: Casa do Psicólogo.

Fontao de Ross, M., \& Mergenthaler, E. (2005). Aplicación del modelo de ciclo terapéutico a la investigación de microprocesos en la psicoterapia de grupo. Revista Argentina de Clínica Psicológica, 14, 53-63.

Gabbard, G. O. (1992). Psiquiatria psicodinâmica na prática clínica. Porto Alegre: Artes Médicas.

Hutz, C. S., \& Nunes, C. H. S. S. (2001). Escala fatorial de ajustamento emocional/neuroticismo. São Paulo: Casa do Psicólogo.

Jacobson, N. S., \& Truax, A. P. (1991). Clinical significance: a statistical approach to defining meaningful change in psychotherapy research. In A. E. Kazdin (Ed.), Methodological issues and strategies in clinical research (2nd ed., pp.521-538). Washington, DC: American Psychological Association.

Laloni, D. T. (2001). Escala de Avaliação de Sintomas-90-RSCL-90-R: adaptação, precisão e validade. Tese de doutorado não-publicada, Pontifícia Universidade Católica de Campinas. Disponível em www.biblioteca digital.puccampinas.edu.br/tde_busca/arquivo.php? codArquivo=29
Lepper, G., \& Mergenthaler, E. (2007). Therapeutic collaboration: how does it work? Psychotherapy Research, $17,576-587$.

Lepper, G., \& Mergenthaler, E. (2008). Observing therapeutic interaction in the "Lisa" case. Psychotherapy Research, 18, 634-644.

Luborsky, L., \& Crits-Christoph, P. (1998). Understanding transference: the CCRT method ( $2^{\text {nd }}$ ed). New York: Basic Books.

Mergenthaler, E. (1996). Emotion-abstraction patterns in verbatim protocols: a new way of describing psychotherapeutic processes. Journal of Consulting and Clinical Psychology, 64, 306-315.

Mergenthaler, E. (2008). Resonating minds: a schoolindependent theoretical conception and its empirical application to psychotherapeutic processes. Psychotherapy Research, 18, 109-126.

Perry, J. C. (1990). Escalas de avaliação dos mecanismos de defesa ( $5^{\mathrm{a}}$ ed.). Manuscrito não-publicado.

Taylor, G. J., Bagby, R. M., \& Parker, J. D. A. (1992). The revised Toronto Alexithymia Scale: some reliability, validity, and normative data. Psychotherapy \& Psychosomatics, 57, 34-41.

Yoshida, E. M. P. (2000). Toronto Alexthymia Scale-TAS: precisão e validade da versão em português. Psicologia: Teoria e Prática, 2 (1), 59-74.

Yoshida, E. M. P. (2008a). Mudança em psicoterapias breves: características de personalidade, conflito interpessoal e estratégia terapêutica. Relatório de Pesquisa, manuscrito não-publicado, Pontifícia Universidade Católica de Campinas.

Yoshida, E. M. P. (2008b). Significância clínica de mudança em processo de psicoterapia psicodinâmica breve. Paidéia, 18 (40), 305-316.

Yoshida, E. M. P. (2009). Modelo dos ciclos terapêuticos: dicionários em português e estudos de validade. Relatório de Pesquisa, manuscrito não-publicado, Pontifícia Universidade Católica de Campinas.

Yoshida, E. M. P., Elyseu Júnior, S., Silva, F. R. C. S., Finotelli Júnior, I., Sanches, F. M., Penteado, E. F., et al. (2009). Psicoterapia psicodinâmica breve: estratégia terapêutica e mudança no padrão de relacionamento conflituoso. Psico USF, 14, 275-285.

Yoshida, E. M. P., \& Rocha, G. M. A. (2007). Avaliação em psicoterapia psicodinâmica. In J. C. Alchieri (Org.), Avaliação psicológica: perspectivas e contextos (pp.237-288). São Paulo: Vetor.

Recebido em: 15/9/2010

Versão final reapresentada em: 10/12/2010

Aprovado em: 3/1/2011 